\title{
On the role of LET-dependent parameters in the determination of the absorbed dose by in-phantom recombination chambers
}

Natalia Golnik, Maciej G. Maciak

\begin{abstract}
The paper discusses the theoretical background in terms of the use of in-phantom recombination chambers in mixed radiation fields, with special attention paid to the question of how the experimentally determined, linear-energy-transfer-dependent (LET) parameters can be applied with regard to the more accurate determination of the chamber response and absorbed dose in mixed radiation fields. Methods of taking the recombination index of radiation quality (RIQ) measurements and theoretical consideration concerning the determination of the absorbed dose are described. Classical Bragg-Gray and Spencer-Attix cavity theories were analysed and their relationship to in-phantom recombination chambers was specified. Methods concerning the estimation of correction factors with regard to RIQ measurements and their importance are highlighted.
\end{abstract}

Keywords: in-phantom recombination chambers $\bullet$ absorbed dose $\bullet$ cavity theory

N. Golnik ${ }^{\bowtie}$, M. G. Maciak

Institute of Metrology and Biomedical Engineering

Warsaw University of Technology

Sw. A. Boboli 8, 02-525 Warsaw, Poland

E-mail: Golnik@mchtr.pw.edu.pl

Received: 14 December 2016

Accepted: 15 December 2017

\section{Introduction}

Recombination chambers were initially defined [1-3] as tissue-equivalent high-pressure ionization chambers, operating under the conditions of the initial recombination of ions in the filling gas. Their unique features and usefulness as mixed-field dose-equivalent meters are based on the exploitation of initial recombination phenomena. As charged particles interact in such a chamber and the gas is ionized, the ions left behind in this process can be collected by the electrodes with the exception of the extent to which they recombine. Initial recombination will depend upon the distance between the ions within the tracks of single particles, as well as upon the applied voltage (which sets the time at which the ions migrate to the electrodes). Thus, for a given voltage, a chamber exhibits more severe recombination in terms of high levels of LET radiation, e.g. neutrons, heavy ions, etc., than for those having low levels of LET radiation, namely electrons, photons, muons and primary high-energy protons, and measuring the amount of recombination (from a saturation curve) makes it possible to experimentally determine a number of LET-dependent parameters of mixed radiation fields [4-7]. An important feature is also that the initial recombination does not depend on the dose rate.

The most common applications of recombination chambers involve the determination of absorbed dose rate, $\dot{D}$, from the value of the saturation current and the so-called recombination index of radiation 
quality (RIQ, $Q_{4}$ - see the description in the next section), which serves as a measurable approximation of the radiation quality factor. Then, the equivalent dose rate, $\dot{H}$, can be calculated as a product of these two quantities:

$$
\dot{H}=\dot{D} \times Q_{4}
$$

Problems associated with the determination of RIQ were described in several earlier papers, e.g. $[4,7-10]$. Much less attention has been paid to the specific aspects of the determination of absorbed dose when using recombination chambers.

Recently, the interest in such measurements has increased, mainly because of the attention paid to neutron doses (and equivalent doses) in terms of $\mathrm{X}$-ray radiotherapy [11-20]. It is also associated with the broader use of proton therapy [21-25] and renewed interest in boron neutron capture therapy (BNCT) [26-29], thus, in the treatment modalities where the recombination chambers can be used for the characterization and monitoring of the beam for quality assurance purposes.

Measurements of the absorbed dose using any ionization chamber start from the experimental determination of the calibration factor which relates the measured saturation current to dosimetric quantities (usually to air kerma in a referenced radiation field). Then, the measurements are performed in a radiation field, which is generally different from the referenced one. Calculation of the absorbed dose in this field involves the use of the earlier determined calibration factor and application of correction factors, which depend on the components and energy of the investigated radiation field. Obviously, such a procedure may introduce a considerable degree of uncertainty in mixed radiation fields of poorly known physical parameters.

The unique features of recombination chambers make it possible to override such a problem, at least partly, taking into account that the sensitivity of the chamber depends on the LET of charged particles that ionize the filling gas. Therefore, some of the correction factors can be estimated from the measured values of RIQ. Such an approach can be especially important in the case of in-phantom ionization chambers, which are mainly used to take measurements where the precise determination of the absorbed dose is required, e.g. in terms of beam dosimetry [30], dosimetric measurements at medical and research facilities [31, 32], or for radiobiological studies [33].

\section{Measurements of RIQ}

The method of determining the recombination index of the radiation quality, denoted by $Q_{4}$, has been broadly discussed in our earlier papers $[4,5,7]$. The main points of the method are:

1) A special voltage, $U_{R}$, that ensures $96 \%$ saturation in the reference field of gamma radiation, has to be determined in the calibration procedure for a certain chamber. Usually, a ${ }^{137} \mathrm{Cs}$ radiation source is used for this purpose.

$$
Q_{4}=\frac{1-f\left(U_{R}\right)}{1-f_{c}\left(U_{R}\right)}=\frac{1-f\left(U_{R}\right)}{1-0.96}=25\left[1-f\left(U_{R}\right)\right]
$$

2) The saturation current and ionization current at the voltage $U_{R}$ should be determined in terms of the radiation field under investigation.

3) $Q_{4}$ is derived from the measured values as:

where $f\left(U_{R}\right)$ and $f_{c}\left(U_{R}\right)$ are the ion collection efficiencies at voltage $U_{R}$ for the investigated type of radiation and for the gamma reference radiation, respectively.

$Q_{4}$ can be evaluated according to the following formula:

$$
Q_{4}=\kappa_{n} Q_{4 n}+\kappa_{\gamma} Q_{4 \gamma}
$$

where $\kappa_{n}$ and $\kappa_{\gamma}$ are the relative contributions of high-LET and low-LET components of the radiation field in terms of the saturation current. In neutron radiation fields where the neutron energy is below $20 \mathrm{MeV}$, the contributions $\kappa_{n}$ and $\kappa_{\gamma}$ can be roughly considered as the neutron and gamma contributions to the total $(n+\gamma)$ kerma in tissue-equivalent (TE) material. $Q_{4 n}$ and $Q_{4 \gamma}$ are the values of the recombination index of radiation quality for neutrons and gamma radiation alone. $Q_{4 \gamma}=1$ by definition.

\section{Determination of the absorbed dose - theoretical considerations}

Determination of the absorbed dose using an ionization chamber always involves calculations of the dose with regard to a medium of interest from the measured value of the dose the gas in the cavity chamber is exposed to.

Theoretical considerations start by first simplifying the assumption that the gas cavity of the chamber is surrounded by material $m$ and the phantom is also composed of a material with an atomic composition and density equal to those of material $m$.

The absorbed dose in the gas in the chamber cavity, $D_{g}$, is by definition expressed as:

$$
D_{g}=\frac{q W}{e \rho V}
$$

where: $q$ is the electrical charge of one sign, generated in the gas cavity of volume $V$; $\rho$ is the gas density, so $\rho V$ is the mass of the gas in the chamber cavity under the conditions the measurements were actually made; $W$ is the mean energy expended on the creation of an ion pair; $e$ is the electron charge.

The electrical charge $q$ can be expressed by the charge $q_{s}$, measured at high polarizing voltage $U_{S}$ (close to saturation for $\gamma$ radiation), and multiplied by appropriate corrections:

$$
q=\frac{q_{s} \Pi k_{q}}{f_{S}}
$$

where $f_{S}=f\left(U_{S}\right)$ is the ion collection efficiency at the polarizing voltage $U_{S}$ and $\Pi k_{q}$ is the product of correction factors which take into account the 
polarization effect, possible lack of linearity of the electrometer, leakage current, and normalization of the recorded figures in terms of the reference monitor values in the case of radiation fields that change over time, etc.

The absorbed dose in terms of the material of the electrodes, $D_{m}$, is:

$$
D_{m}=D_{g} r_{m g}
$$

where $r_{m g}$ is the conversion factor.

The value of $r_{m g}$ depends on the size of the cavity and radiation energy (or radiation quality). According to the Bragg-Gray cavity theory there are two extreme cases with regard to "small" and "large" detectors.

The most common approach for the determination of the absorbed dose of a medium using in-phantom ionization chambers, also recombination ones, involves the assumption that the gas cavity fulfils the conditions of the so-called Bragg-Gray small detector. A cavity is regarded to be so small that:

- the energy imparted to the cavity from electrons released by photons in the cavity is negligible compared to the energy imparted from electrons released by photons in the surrounding medium that pass through the cavity,

- the cavity should not disturb the fluence of electrons in the medium, i.e. the fluence of electrons traversing the cavity is assumed to be identical to that existing at the point of interest in the medium in the absence of the cavity.

The practical argument made by Gray in the early development of the theory was that as long as the ionization in the gas of the cavity increased linearly with the size of the cavity, the cavity could be considered to fulfil the requirements of a Bragg-Gray detector.

Under such conditions, the ratio of the dose in the medium to dose in the detector (gas) can be expressed as:

$$
r_{m g}=S_{m g}=\frac{\left(\frac{\bar{S}}{\rho}\right)_{\mathrm{med}}}{\left(\frac{\bar{S}}{\rho}\right)_{\text {gas }}}
$$

where: $S_{m g}$ is the ratio of the mean mass collision stopping power, $(\bar{S} / \rho)_{\text {med }}$, of the secondary charged particles in the material, $m$, to those in the gas.

While many ionization chambers can be considered as being Bragg-Gray detectors, the experimental data showed that the measured ionization current was significantly different from the one calculated according to Eq. (7) when the material of the wall possessed a high atomic number for small cavities. The deviation from Bragg-Gray cavity theory was explained by Spencer and Attix [34], who extended the Bragg-Gray cavity theory taking into consideration the delta particles. All electrons, including delta electrons with energies above a cut-off energy, $\Delta$, were included in the fluence spectrum, while electrons with energies below $\Delta$ were assumed to be locally absorbed in the cavity. A new expression for the conversion factor $D^{\text {med }} / D^{\text {gas }}$ was formulated as:

$$
\frac{D^{\text {med }}}{D^{\text {gas }}}=\frac{\int_{\Delta}^{E_{\max }} \Phi_{\mathrm{E}}^{\delta}(\mathrm{E})\left(\frac{S_{\Delta}}{\rho}\right)_{\text {med }} d \mathrm{E}+\mathrm{TE}^{\text {med }}}{\int_{\Delta}^{E_{\max }} \Phi_{\mathrm{E}}^{\delta}(\mathrm{E})\left(\frac{S_{\Delta}}{\rho}\right)_{\text {gas }} d \mathrm{E}+\mathrm{TE}^{\text {gas }}}
$$

The electron fluence, $\Phi_{\mathrm{E}}^{\delta}$, in Eq. (8) now includes primary electrons, as well as knock-on electrons with energies above $\Delta$. The integrals go from $\Delta$ to the maximum energy value and the restricted mass stopping powers, $\left(S_{\Delta} / \rho\right)$, are used instead of the unrestricted ones. The terms $\mathrm{TE}^{\mathrm{med}}$ and $\mathrm{TE}^{\mathrm{gas}}$ are the so-called track-ends terms [35], which correct for the energy deposition of the electrons that possess energies less than $\Delta$.

The value of the restriction $\Delta$ is correlated with the mean chord length of the gas cavity and traditionally regarded as the energy of an electron whose range is equal to the mean path length in the cavity. In planar chambers the range of energies of the electrons in the gas is twice the distance between the electrodes $(2 d$, where $d$ is the distance between the electrodes expressed in units of mass per unit area). For typical ionization chambers, with a cavity of a few millimetres, the value of $\Delta$ is often set arbitrarily at $10 \mathrm{keV}$. Fortunately, $D^{\mathrm{med}} / D^{\text {gas }}$ is a slowly changing function of $\Delta$ and in practical situations the theory is relatively insensitive to changes in $\Delta$.

A more qualified way of choosing the energy limit was discussed by Spencer [36]. Some of the $\delta$ electrons generated in the cavity of energies below $\Delta$ will deposit part of their energy outside of the cavity. Similarly, some of the $\delta$ electrons generated in the cavity with energies larger than $\Delta$ will deposit part of their energies inside the cavity although they are considered not to contribute to the "locally" absorbed energy. By choosing the appropriate $\Delta$, the energy carried out of the cavity by $\delta$ electrons generated with energies larger than $\Delta$ could be exactly compensated for by the energy deposited in the cavity from $\delta$ electrons generated outside of the cavity with energies larger than $\Delta$.

To calculate the stopping power ratios from Eq. (8), Monte Carlo methods are typically used. However, in many situations where the difference in the atomic compositions of the medium and gas inside the cavity is small, mean values of the restricted stopping power can be used as a good approximation. Then,

$$
r_{m g}=\left(S_{m g}\right)_{\Delta}=\frac{\left(\frac{\bar{S}_{\Delta}}{\rho}\right)_{m}}{\left(\frac{\bar{S}_{\Delta}}{\rho}\right)_{g}}
$$

Practically, the approximation of Eq. (9) can be used for most in-phantom tissue-equivalent recombination chambers over a broad energy range, even for chambers filled with a gas with an atomic composition that slightly differs from those of the walls.

The absorbed dose in the material, $t$, is different to that of material, $m$, (e.g. water, or tissues with atomic compositions considerably different 
from the TE material of the electrodes) and can be expressed as:

$$
D_{t}=D_{m} r_{t m}
$$

where $r_{t m}$ is the conversion factor from material $m$ to material $t$.

Finally, by taking into account Eqs. (4), (5) and (6), the absorbed dose in the material of the phantom is given by Eq. (11):

$$
D_{t}=r_{t m} r_{m g} \frac{q_{S} \Pi k_{q} W}{f_{S} e \rho V}
$$

For the situations when the approximation of Eq. (9) is valid, the above equation can be written as:

$$
D_{t}=r_{t m} \frac{\left(S_{m g}\right)_{\Delta} q_{S} \Pi k_{q} W}{f_{S} e \rho V}
$$

If the composition of material $t$ is (dosimetrically) not very different from material $m$, then the approximation:

$$
r_{t m}=\left(S_{t m}\right)_{\Delta}
$$

can be used and Eq. (12) transformed into the form:

$$
D_{t}=\frac{\left(S_{t g}\right)_{\Delta} q_{S} \Pi k_{q} W}{f_{S} e \rho V}
$$

where

$$
\left(S_{t g}\right)_{\Delta}=\frac{\left(\frac{\bar{S}_{\Delta}}{\rho}\right)_{t}}{\left(\frac{\bar{S}_{\Delta}}{\rho}\right)_{g}}
$$

is the ratio of the mean restricted mass stopping powers of the charged particles in material $t$ and in the gas. Again, the value of the restriction $\Delta$ is not crucial and can be related to the thickness of the material layer in which the absorbed dose is averaged. Usually, the ratio of unrestricted stopping power values can be used for this purpose. The possible impact of approximations (9) and (13) can be included in the production of correction factors $k_{q}$.

The second extreme case, in relation to the "small" gas cavity, is the situation of the chambers with large cavities, when the gas is ionized by secondary charged particles generated in the cavity and the contribution from the particles generated in the walls is negligibly small. Then, $r_{m g}$ is expressed as:

$$
\begin{array}{ll}
r_{m g}=\mu_{m g}=\frac{\left(\mu_{e n}\right)_{m}}{\left(\mu_{e n}\right)_{g}} & \text { for photons } \\
r_{m g}=K_{m, g}=\frac{K_{m}}{K_{g}} & \text { for neutrons }
\end{array}
$$

where $\left(\mu_{e n}\right)_{m}$ and $\left(\mu_{e n}\right)_{g}$ are photon mass energy transfer coefficients in material $m$ and in the gas, respectively. $K_{m}$ and $K_{g}$ are the values of specific kerma.

In all intermediate situations, when neither the conditions of small nor large detectors are applicable, the combination of the values of $S_{m g}$ and $K_{m g}$ should be applied. For fast neutrons with energies below $20 \mathrm{MeV}$ and planar chambers, $r_{m g}$ can be approximated by Eq. (17) [37]

$$
r_{m g}=\left(\frac{1}{K_{m g}} \frac{3 d}{3 d+R}+\frac{1}{S_{m g}} \frac{R}{3 d+R}\right)^{-1}
$$

where, $d$ is the distance between the electrodes (in units of mass per unit area) and $R$ is the mean (dose averaged) range of the secondary charged particles (in units of mass per unit area).

The above considerations concern the situation when the gas cavity, filled with TE gas, is surrounded by one type of material $m$. In practice, there are always a few layers. In TE chambers, the first layer is made of tissue equivalent material, then there is an insulation layer (usually polytetrafluoroethylene or polyethylene) and finally the housing (usually aluminium but also other materials have been recently used, e.g. titanium). In such cases, calculating $r_{m g}$ would require all the layers and their atomic compositions to be taken into account. The possible solution to this problem is numerical modelling of the radiation transport. Another approach is based on the experimental determination of the so-called wall coefficients for a particular detector [37]. In the case of organic materials, even when surrounded by reasonably thick aluminium housing, the wall coefficients are usually very similar, especially in the case of high energy radiation [38]. Therefore, Eq. (14) can be usually used in practice with the product of correction factors being very similar.

\section{Calibration of the chambers and correlations between chamber sensitivity and RIQ}

Equation (14) includes the mass of the gas in the chamber cavity $(\rho V)$. The direct determination of the cavity volume and gas density is always associated with relatively large uncertainties, so it is determined indirectly in the process of calibration with regard to a reference gamma radiation field. In our measurements, the ${ }^{137} \mathrm{Cs}$ isotopic radiation source is used and calibration is performed free-in-air. The thickness of the chamber wall is sufficient to achieve a charged-particle equilibrium, so there is no need to use any special cups. The reference quantity, in most cases, is air kerma, $K_{a}$. In order to determine the absorbed dose in material $m$, the attenuation of the primary radiation in the chamber's wall and also scattering by the elements of the chamber in the layer directly adjacent to the gas cavity should be taken into account. Other relationships between the collected charge and absorbed dose for the reference radiation are the same as was generally considered in the previous chapter. Therefore, the sensitivity of the chamber to the reference radiation, $A$, defined as the ratio of the electrical charge generated in the gas cavity to the absorbed dose in the material adjacent to the gas cavity, can be expressed as:

$$
A=\left(\frac{q}{D_{m}}\right)_{c}=\left(\frac{q_{s} \Pi k_{q}}{f_{S} K_{m} k_{a, r}}\right)_{c}=\left(\frac{q_{s} \Pi k_{q}}{f_{S} K_{a} \mu_{m a} k_{a, r}}\right)_{c}
$$


where: $K_{m}$ is the kerma of the reference radiation in material $m$, introduced here in order to show the transformation of the formula; $\mu_{m a}$ is the ratio of mass energy transfer coefficients in material $m$ and in air; $k_{a, r}$ is a coefficient which accounts for attenuation and scattering; index $c$ indicates that the equation concerns calibration in the field of the reference radiation.

The mass $(\rho V)$ of the gas can be derived from the experimentally measured sensitivity, using relationship (19):

$$
A=\left(\frac{q}{D_{m}}\right)_{c}=\left(\frac{q}{D_{g}\left(S_{m g}\right)_{\Delta}}\right)_{c}=\left(\frac{e \rho V}{D_{g}\left(S_{m g}\right)_{\Delta}}\right)_{c}
$$

By combining Eqs. (14) and (19), the absorbed dose in phantom material $t$ can be expressed as:

$$
D_{t}=\frac{q_{S} \Pi k_{q}}{f_{S} A} \frac{\left(\frac{\bar{S}_{\Delta}}{\rho}\right)_{t}}{\left(\frac{\bar{S}_{\Delta}}{\rho}\right)_{m}} \frac{(\rho V)_{c}}{\rho V} \frac{W}{W_{c}} \frac{\left(S_{m g}\right)_{\Delta}}{\left(S_{m g}\right)_{\Delta c}}
$$

It is worth underlining that two final factors in Eq. (20), i.e. $W / W_{c}$ and $\left(S_{m g}\right)_{\Delta} /\left(S_{m g}\right)_{\Delta c}$, depend on the energy of the charged particles and can be correlated with the recombination index of radiation quality $Q_{4}$. The correlation in terms of $W / W_{c}$ can be expressed as [8]:

$$
\frac{W}{W_{c}}=1+\beta_{W}\left(Q_{4}-1\right)
$$

where, $\beta_{W}=0.008$ [8] for practically the entire energy range of charged particles and over a broad range of neutron energies (with the exception of epithermal neutrons, if the chamber is irradiated without phantom).

For high energy neutrons, there are also two other approximate correlations [39]:

$$
r_{m g}=1-0.009\left(Q_{4 n}-9.5\right)
$$

where $k_{S}=1.137$ and $\beta_{S}=0.006$ [39], and:

$$
\left(\bar{S}_{\text {water/air }}\right)_{\Delta}=k_{S}\left(1+\beta_{S}\left(Q_{4}-1\right)\right)
$$

where $Q_{4 n}$ is the recombination index of radiation quality for neutrons alone.

\section{Discussion and summary}

The approximate value of the total absorbed dose measured by an ionization chamber (also by a recombination one) is given by the ratio $q_{s} / A$. The values of other factors of Eq. (18) are included as the ratios of similar quantities, so their values are almost identical. By taking into account Eq. (18), one can also see that the first term in Eq. (20) contains the ratios of $\left(f_{S}\right)_{c} / f_{S}$ and $\Pi k_{q} /\left(\Pi k_{q}\right)_{c}$, which can usually be estimated more accurately than the absolute values of corresponding physical quantities. Some of the correction factors, $k_{q}$, may be reduced, since they are the same in the numerator and denom- inator of the fraction (see the description of Eq. (5) and Eqs. (18) and (19)). This concerns, for example, the absolute values of the electrometer readings and other factors dependent on the measuring system.

In terms of neutron radiation, the ratio $S_{t} / S_{m}$ can be correlated over a rather broad energy range with the content of hydrogen in materials $t$ and $m$. The ratio $(\rho V)_{c} / \rho V$ can be influenced by possible leakage of the gas during the period between calibration and the recording of measurements and by gas adsorption/desorption in the elements of the chamber due to changes in temperature.

It should also be kept in mind that the assumptions of Eq. (20) are not always fulfilled. This concerns, for example, fast neutrons with energies below a few MeV. In this case, the ratio of stopping powers should be replaced by the conversion factor $r_{m g}$, which strongly depends on the percentage of hydrogen in the gas of the chamber. Some of the recombination chambers are intentionally filled with a gas containing more hydrogen rather than with the standard TE material. For such chambers, which are used in mixed gamma-neutron radiation fields, it is often more accurate to determine the absorbed dose simply from the ratio $q_{s} / A$ than to use Eq. (20) which requires the complex calculation of the ratios of correction factors that by and large compensate each other.

The relationships presented in this paper are useful in most cases, but such measuring conditions can also exist where additional corrections are needed. An example of such a case, which is not considered here, is the situation when the radiation field in the phantom possesses a large gradient in terms of the dose and is considerably distorted by the gas cavity. The displacement factor should then be introduced, which accounts for the shift of the measuring point from the geometrical centre of the chamber in the direction of the gradient of radiation intensity.

Generally, recombination methods allow for the determination of the absorbed dose and dose equivalents in phantoms irradiated in mixed radiation fields of non-limited composition and energy range. Nevertheless, to obtain precise measurements the correction factors and uncertainty of the absorbed dose have to be specifically taken into consideration under all measuring conditions.

Acknowledgment. This paper was partly supported by the National Science Centre in Poland under grant no. 2015/19/N/ST7/01202.

\section{References}

1. Zielczyński, M. (1962). Use of columnar recombination for determination of relative biological efficiency of radiation. Nukleonika, 7, 175-182 (in Russian).

2. Zielczyński, M. (1963). Recombination method for determination of linear energy transfer of mixed radiation. In Symposium on Neutron Detection, Dosimetry, and Standardization, 10-14 December 1962 (pp. 397-404). Vienna, Austria: International Atomic Energy Agency. 
3. Sullivan, A. H., \& Baarli, J. (1963). An ionization chamber for the estimation of the biological effectiveness of radiation. Geneva, Switzerland: CERN. (Report 63-17).

4. Zielczyński, M., Golnik, N., Makarewicz, M., \& Sullivan, A. H. (1981). Definition of radiation quality by initial recombination of ions. In 7 th Symposium on Microdosimetry, 8-12 September 1980 (pp. 853-862). Oxford, United Kingdom: Harwood Academic Publishers.

5. Zielczyński, M., \& Golnik, N. (1994). Recombination index of radiation quality - measuring and applications. Radiat. Prot. Dosim., 52, 419-422.

6. Golnik, N. (1995). Microdosimetry using a recombination chamber: Method and applications. Radiat. Prot. Dosim., 61(1/3), 125-128.

7. Golnik, N. (1996). Recombination methods in the dosimetry of mixed radiation. Swierk, Poland: Institute of Atomic Energy. (Report IAE-20/A).

8. Zielczyński, M., \& Golnik, N. (1994). Energy expended to create an ion pair as a factor dependent on radiation quality. In International Symposium on Measurement Assurance in Dosimetry, 24-27 May 1993 (pp. 383-391). Vienna, Austria: International Atomic Energy Agency.

9. Silari, M., Agosteo, S., Beck, P., Bedogni, R., Cale, E., Caresana, M., Domingo, C., Donadille, L., Dubourg, N., Esposito, A., Fehrenbacher, G., Fernandez, F., Ferrarini, M., Fiechter, A., Fuchs, A., Garcia, M. J., Golnik, N., Gutermuth, F., Khurana, S., Klages, Th., Latocha, M., Mares, V., Mayer, S., Radon, T., Reithmeier, H., Rollet, S., Roos, H., Ruhm, W., Sandri, S., Schardt, D., Simmer, G., Spurny, F., Trompier, F., Villa-Grasa, E., Weitzenegger, E., Wiegel, B., Wielunski, M., Wissmann, F., Zechner, A., \& Zielczynski, M. (2009). Intercomparison of radiation protection devices in a high-energy stray neutron field. Part III: Instrument response. Radiat. Meas., 44(7/8), 673-691. DOI: 10.1016/j.radmeas.2009.05.005.

10. Caresana, M., Denker, A., Esposito, A., Ferrarini, M., Golnik, N., Hohmann, E., Leuschner, A., LuszikBhadra, M., Manessi, G., Mayer, S., Ott, K., Roehrich, J., Silari, M., Trompier, F., Volnhals, M., \& Wielunski, M. (2014). Intercomparison of radiation protection instrumentation in a pulsed neutron field. Nucl. Instrum. Methods Phys. Res. Sect. A-Accel. Spectrom. Dect. Assoc. Equip., 737, 203-213. DOI: 10.1016/j. nima.2013.11.073.

11. Miljanic, S., Bordy, J. -M., d'Errico, F., Harrison, R., \& Olko, P. (2014). Out-of-field dose measurements in radiotherapy - An overview of activity of EURADOS Working Group 9: Radiation protection in medicine. Radiat. Meas., 71, 270-275. DOI: 10.1016/j.radmeas.2014.04.026.

12. Kaderka, R., Schardt, D., Durante, M., Berger, T., Ramm, U., Licher, J., \& La Tessa, C. (2012). Outof-field dose measurements in a water phantom using different radiotherapy modalities. Phys. Med. Biol., 57(16), 5059-5074. DOI: 10.1088/00319155/57/16/5059.

13. Hälg, R. A., Besserer, J., Boschung, M., Mayer, S., Lomax, A. J., \& Schneider, U. (2014). Measurements of the neutron dose equivalent for various radiation qualities, treatment machines and delivery techniques in radiation therapy. Phys. Med. Biol., 59(10), 2457-2468. DOI: $10.1088 / 0031-9155 / 59 / 10 / 2457$.

14. Sanchez-Doblado, F., Domingo, C., Gomez, F., Sánchez-Nieto, B., Muñiz, J. L., García-Fusté, M. J., Expósito, M. R., Barquero, R., Hartmann, G., Terrón, J. A., Pena, J., Méndez, R., Gutiérrez, F., Guerre, F.
X., Roselló, J., Núñez, N., Brualla-González, L., Manchado, F., Lorente, A., Gallego, E., Capote, R., Planes, D., Lagares, J. I., González-Soto, X., Sansaloni, F., Colmenares, R., Amgarou, K., Morales, E., Bedogni, R., Cano, J. P., \& Fernández, F. (2012). Estimation of neutron-equivalent dose in organs of patients undergoing radiotherapy by the use of a novel online digital detector. Phys. Med. Biol., 57(19), 6167-6191. DOI: 10.1088/0031-9155/57/19/6167.

15. Irazola, L., Lorenzoli, M., Bedogni, R., Pola, A., Terron, J. A., Sanchez-Nieto, B., Exposito, M. R. Lageras, J. I., Sansaloni, F., \& Sanchez-Doblado, F. (2014). A new online detector for estimation of peripheral neutron equivalent dose in organ. Med. Phys., 41(11), art. no. 112105. DOI: 10.1118/1.4898591.

16. Haelg, R. A., Besserer, J., Boschung, M., SánchezNieto, B., Muñiz, J. L., García-Fusté, M. J., Expósito, M. R., Barquero, R., Hartmann, G., Terrón, J. A., Pena, J., Méndez, R., Gutiérrez, F., Guerre, F. X., Roselló, J., Núñez, L., Brualla-González, L., Manchado, F., Lorente, A., Gallego, E., Capote, R., Planes, D., Lagares, J. I., González-Soto, X., Sansaloni, F., Colmenares, R., Amgarou, K., Morales, E., Bedogni, R., Cano, J. P., \& Fernández, F. (2014). Measurements of the neutron dose equivalent for various radiation qualities, treatment. Phys. Med. Biol., 59(10), 2457-2468. DOI: 10.1088/0031-9155/59/10/2457

17. Di Fulvio, A., Domingo, C., De San Pedro, M., D'Agostino, E., Caresana, M., Tana, L., \& d'Errico, F. (2013). Superheated emulsions and track etch detectors for photoneutron measurements. Radiat. Meas., 57, 19-28. DOI: 10.1016/j.radmeas.2013.11.004.

18. Konefal, A., Orlef, A., \& Bieniasiewicz, M. (2016). Measurements of neutron radiation and induced radioactivity for the new medical linear accelerator, the Varian TrueBeam. Radiat. Meas., 86, 8-15. DOI: 10.1016/j.radmeas.2015.12.039.

19. Kowalik, A., Jackowiak, W., Malicki, J., Skórska, M., Adamczyk, M., Konstanty, E., Piotrowski, T., \& Polaczek-Grelik, K. (2017). Measurements of doses from photon beam irradiation and scattered neutrons in an anthropomorphic phantom model of prostate cancer: a comparison between 3DCRT, IMRT and tomotherapy. Nukleonika, 62(1), 29-35. DOI: 10.1515/ nuka-2017-0005.

20. Romero-Exposito, M., Domingo, C., Sanchez-Doblado, F., Ortega-Gelabert, O., \& Gallego, S. (2016). Experimental evaluation of neutron dose in radiotherapy patients: Which dose? Med. Phys., 43(1), 360-367. DOI: $10.1118 / 1.4938578$.

21. Particle Therapy Co-Operative Group (July, 2017). Particle Therapy Centers. Retrieved August 08, 2017 from https://www.ptcog.ch/index.php/facilities-inoperation, https://www.ptcog.ch/index.php/facilitiesunder-construction.

22. IFJ Cyclotron Centre Bronowice. (2015). Cyclotron Centre Bronowice. Retrieved August 08, 2017, from https://ccb.ifj.edu.pl/en.home.html.

23. Brenner, D. J., \& Hatt, E. J. (2008). Secondary neutrons in clinical proton radiotherapy: A charged issue. Radiother. Oncol., 86(2), 165-170. DOI: 10.1016/j.radonc.

24. Farah, J., Mares, V., Romero-Exposito, M., Trinkl, S., Domingo, C., Dufek, V., Klodowska, M., Kubancak, J., Knezevic, Z., Liszka, M., Majer, M., Miljanic, S., Ploc, O., Schinner, K., Stolarczyk, L., Trompier, F., Wielunski, M., Olko, P., \& Harrison, R. M. (2015). Measurement of stray radiation within a scanning proton therapy facility: EURADOS WG9 intercomparison exercise of active dosimetry systems. Med. Phys., 42(5), 2572-2584. DOI: $10.1118 / 1.4916667$. 
25. Schneider, U., \& Haelg, R. (2015). The impact of neutrons in clinical proton therapy. Frontiers in Oncology, 5, art. no. 235. DOI: 10.3389/fonc.2015.00235.

26. Kumada, H., Matsumura, A., Sakurai, H., Sakae, T., Yoshioka, M., Kobayashi, H., Matsumoto, H., Kiyanagi, Y., Shibata, T., \& Nakashima, H. (2014). Project for the development of the linac based NCT facility in University of Tsukuba. Appl. Radiat. Isot., 88, 211-215. DOI: 10.1016/j.apradiso.2014.02.018.

27. Takada, K., Kumada, H., Isobe, T., Terunuma, T., Kamizawa, S., Sakurai, H., Sakae, T., \& Matsumura, A. (2015). Whole-body dose evaluation with an adaptive treatment planning system for boron neutron capture therapy. Radiat. Prot. Dosim., 167(4), 584-590. DOI: 10.1093/rpd/ncu357.

28. Durisi, E., Alikaniotis, K., Borla, O., Bragato, F., Costa, M., Giannini, G., Monti, V., Visca, L., Vivaldo, G., \& Zanini, A. (2015). Design and simulation of an optimized e-linac based neutron source for BNCT research. Appl. Radiat. Isot., 106, 63-67. DOI: 10.1016/j.apradiso.2015.07.039.

29. Miyatake, S. I., Kawabata, S., Hiramatsu, R., Kuroiwa, T., Suzuki, M., Kondo, N., \& Ono, K. (2016). Boron neutron capture therapy for malignant brain tumors. Neurol. Med. Chir., 56(7), 361-371. DOI: 10.2176/nmc.ra.2015-0297.

30. Zielczyński, M., Komochkov, M. M., Sychev, B. S., \& Cherevatenko, A. P. (1968). Measurements of the quality factor for high energy protons in the water phantom. Nukleonika, 13(2), 165-170.

31. Golnik, N., Cherevatenko, E. P., Serov, A. Y., Shvidkij, S. V., Sychev, B. S., \& Zielczyński, M. (1997). Recombination index of radiation quality of medical high energy neutron beams. Radiat. Prot. Dosim., $70(1 / 4), 215-218$.
32. Golnik, N., Zielczyński, M., Bulski, W., Tulik, P., \& Pałko, T. (2007). Measurements of the neutron dose near a $15 \mathrm{MV}$ medical linear accelerator. Radiat. Prot. Dosim., 126(1/4), 619-622. DOI: 10.1093/ $\mathrm{rpd} / \mathrm{ncm} 125$.

33. Golnik, N., Gryziński, M. A., Kowalska, M., Meronka, K., \& Tulik, P. (2014). Characterization of radiation field for irradiation of biological samples at nuclear reactor - comparison of twin detector and recombination methods. Radiat. Prot. Dosim., 161(1/4), 196-200. DOI: $10.1093 / \mathrm{rpd} / \mathrm{nct} 341$.

34. Spencer, L. V., \& Attix, F. H. (1955). A theory of cavity ionization. Radiat. Res., 3(3), 239-254.

35. Nahum, A. E. (1978). Water/air mass stopping power ratios for megavoltage photon and electron beams. Phys. Med. Biol., 23, 24-38.

36. Spencer, L. V. (1971). Remarks on the theory of energy deposition in cavities. Acta Radiol. Ther. Phys. Biol., 10(1), 1-20.

37. Zielczyński, M. (1988). Technique of determining dose in medical beams of high energy particles. Dubna, Russia: JINR. (JINR Commun. R16-88-531). (in Russian).

38. Golnik, N., \& Zielczyński, M. (1997). Dosimetry of neutron beams with energy of hundreds of MeV. In International Conference Neutrons in Research and Industry, June 9, 1996 (pp. 254-263). Crete, Greece: International Society for Optics and Photonics SPIE.

39. Zielczyński, M., \& Golnik, N. (1999). Dosimetry of TRIGA reactor fields using high pressure ionization chambers. Swierk, Poland: Institute of Atomic Energy. (Report IAE-61/A). 